\title{
Calcium Regulation of Neurite Elongation and Growth Cone Motility
}

\author{
Mark P. Mattson and Stanley B. Kater \\ Program in Neuronal Growth and Development, Department of Anatomy, Colorado State University, Fort Collins, Colorado \\ 80523
}

\begin{abstract}
Neurite outgrowth from isolated, identified molluscan (Helisoma trivolvis) neurons in culture can be suppressed by neurotransmitters and electrical activity, both of which increase intraneuronal $\mathrm{Ca}^{2+}$ levels (Haydon et al., 1984; Cohan et al., 1986, 1987). We explored the possibility of a causal relationship between $\mathrm{Ca}^{2+}$ influx from the cell exterior and neurite outgrowth using a spectrum of pharmacological manipulations known to affect transmembrane $\mathrm{Ca}^{2+}$ flux. $\mathrm{Ca}^{2+}$ ionophore A23187, an agent expected to increase $\mathrm{Ca}^{2+}$ influx, suppressed both elongation and motile growth cone structures (i.e., filopodia and lamellipodia) in a dose-dependent $\left(10^{-8}-10^{-6} \mathrm{M}\right)$ and reversible manner. Furthermore, high concentrations of $\mathrm{Ca}^{2+}$ channel blockers $\left(\mathrm{La}^{3+}, \mathrm{Cd}^{2+}, \mathrm{Co}^{2+}\right.$; e.g., $10^{-4} \mathrm{M} \mathrm{La}{ }^{3+}$ ) suppressed both elongation and growth cone movements. These data support previous experiments, which indicated that neurite outgrowth is dependent upon a specific range of intracellular $\mathrm{Ca}^{2+}$ concentrations (Connor, 1986; Cohan et al., 1987). However, tests of the dose-dependency of the effects of $\mathrm{Ca}^{2+}$ channel blockers on outgrowth revealed that specific, low concentrations of $\mathrm{Ca}^{2+}$ channel blockers (e.g., $1^{-5} \mathrm{M} \mathrm{La^{3+ }}$ ) caused, simultaneously, a reduction of growth cone filopodia and an acceleration of elongation. Consistent with the results using low levels of $\mathrm{Ca}^{2+}$ channel blockers, reduced extracellular $\mathrm{Ca}^{2+}$-stimulated neurite elongation while suppressing growth cone motility. Finally, neurotransmitter regulation of neurite outgrowth was shown to require influx of extracellular $\mathrm{Ca}^{2+}$; serotonin inhibition of neuron $\mathrm{B} 19$ was prevented by $\mathrm{La}^{3+}\left(10^{-5} \mathrm{M}\right)$ or by incubation in a reduced $\mathrm{Ca}^{2+}$ environment. Taken together, these results indicate that there are optimum levels of $\mathrm{Ca}^{2+}$ influx that promote normal neurite elongation and growth cone movements; these 2 components of outgrowth appear to have differential sensitivities to $\mathrm{Ca}^{2+}$.
\end{abstract}

The biochemical basis of neuronal outgrowth has been an area of intense investigation. The pioneering work on nerve growth factor led the way in uncovering a variety of environmental conditions and specific molecules that regulate the initiation of neuronal outgrowth (e.g., Levi-Montalcini and Angeletti, 1968; Schubert et al., 1978; Greene and Shooter, 1980; Gundersen

\footnotetext{
Received Jan. 29, 1987; revised Apr. 16, 1987; accepted Apr. 22, 1987

The authors acknowledge the technical assistance of Michelle Mischke, Denise Dehnbostel, and Lisa Spirio. Special thanks to Drs. C. Cohan, J. Connor, J. Goldberg, P. Guthrie, P. I Iaydon, and D. McCobb for their critical comments on the manuscript. This work was supported by NIH Grants NS 08054 (M.P.M.); NS 24683, NS 24561, and NS 15350 (S.B.K.); and a gift from the Monsanto Corporation.

Correspondence should be addressed to Dr. Mark P. Mattson at the above address.

Copyright (C) 1987 Society for Neuroscience $0270-6474 / 87 / 124034-09 \$ 02.00 / 0$
}

and Barrett, 1980). The cellular mechanisms of action of the regulators of neuronal outgrowth are not clear, but second messengers, including cAMP (Schubcrt ct al., 1978; Nirenberg et al., 1984), calcium (Schubert et al., 1978; Connor, 1986; Cohan et al., 1986, 1987), and inositol phospholipids (Ishii, 1978; Spinelli and Ishii, 1983; Hama et al., 1986) are implicated. $\mathrm{Ca}^{2+}$ affects the assembly states of both microtubules (Schliwa et al., 1981) and microfilaments (Adelstein and Eisenberg, 1980) and is a prime candidate as an intracellular regulator of neurite elongation and motile growth cone structures (filopodia and lamellipodia).

In understanding the genesis of functional neuronal architecture, the regulation of cessation of neuronal outgrowth is perhaps of equal importance to the promotion of outgrowth. Little knowledge has been available on the specific chemical mediators that stabilize previously growing neurites. We recently described 2 independent signals that inhibit neuronal outgrowth: neurotransmitters from exogenous sources (Haydon et al., 1984) and electrical activity (Cohan and Kater, 1986). These findings were possible, in part, because of the ability to culture isolated, identified neurons of the mollusk Helisoma (see Kater, 1985, for a review). The presence of specific neurotransmitters can profoundly alter neuronal outgrowth. Serotonin, for instance, can inhibit the neurite elongation and growth cone movements of particular identified neurons, while not affecting other specific neurons (Haydon ct al., 1984). While neurotransmitters act on defined subsets of neurons, we have found that a second regulatory variable, the generation of action potentials, acts on all tested neurons to abruptly terminate filopodial and lamellipodial activities and neurite elongation (Cohan and Kater, 1986).

Several authors have suggested a role for $\mathrm{Ca}^{2+}$ in the regulation of neurite outgrowth (Schubert et al., 1978; Llinas, 1979; Gundersen and Barrett, 1980; Anglister et al., 1982; Kostenko et al., 1983; Bixby and Spitzer, 1984), and this second messenger system was thus an obvious candidate for direct measurement. Connor's (1986) initial fura-2 studies on cultured mammalian CNS neurons gave the first substantive data on intracellular $\mathrm{Ca}^{2+}$ and outgrowth. Cohan et al. $(1986,1987)$ were then able to measure intracellular $\mathrm{Ca}^{2+}$ in neurons exposed to substances and conditions known to affect outgrowth (serotonin or induced action potentials); they demonstrated strong correlations between the level of free cytoplasmic $\mathrm{Ca}^{2+}$ and the growth status of the neuron. Their data indicated that an optimal level of free intracellular $\mathrm{Ca}^{2+}$ was required for outgrowth to continue.

The present study further tests a "calcium hypothesis" of control of neurite outgrowth by direct manipulation of $\mathrm{Ca}^{2+}$ flux across the plasma membrane. Standard pharmacological agents known for their particular effects on $\mathrm{Ca}^{2+}$ were employed to help establish a causal relationship between $\mathrm{Ca}^{2+}$ influx from the cell 
exterior and neurite outgrowth. Since the pharmacology of $\mathrm{Ca}^{2+}$ can be capricious, we used a spectrum of agents and found quite consistent results using $\mathrm{Ca}^{2+}$ ionophore $\mathrm{A} 23187, \mathrm{Ca}^{2+}$ channel blockers $\left(\mathrm{La}^{3+}, \mathrm{Co}^{2+}\right.$, and $\left.\mathrm{Cd}^{2+}\right)$, and reduced or zero $\mathrm{Ca}^{2+}$ medium. The combined results of the experiments reported here demonstrate that motile growth cone structures, as well as neurite elongation, are regulated by $\mathrm{Ca}^{2+}$ influx. However, the optimal levels of $\mathrm{Ca}^{2+}$ influx required by these 2 components of outgrowth appear to differ, a finding that allows further definition of the roles of growth cones in the generation of neuronal architecture.

\section{Materials and Methods}

Animal dissection and neuronal culture. Adult snails of Helisoma trivolvis (red) with shell diameters of $10-18 \mathrm{~mm}$ were maintained as previously described (Haydon et al., 1985). Procedures for dissections, buccal ganglia removal, and identified neuron isolation have been described previously (Wong et al., 1981; Haydon et al., 1985). Isolated neurons were transferred to culture dishes with a polylysine-coated glass or plastic substratum, containing $2 \mathrm{ml}$ of defined HL-15 medium (Gibco, Grand Island, NY) conditioned with Helisoma brains [conditioned medium contains a factor that associates with the substratum and promotes extensive neurite outgrowth (Wong et al., 1981, 1984)]. Neurons were cultured in a humidified room air atmosphere at $22-25^{\circ} \mathrm{C}$.

Treatment of neuronal cultures. All experiments were conducted on growing identified buccal neurons B19 and B5 at 20-24 hr postplating. Ionophore A23187, nifedipine, and verapamil were dissolved in dimethyl sulfoxide (DMSO) and were added to cultures in 5-10 $\mu$ l volumes (DMSO alone at these levels was added to control plates and did not affect outgrowth parameters). $\mathrm{LaCl}_{3}, \mathrm{CoCl}_{2}, \mathrm{CdCl}_{2}$, sodium dantrolene, and serotonin were dissolved in HL-15 and added to cultures in 20 $200 \mu \mathrm{l}$ volumes. All concentrations reported are the final concentrations in culture medium. All agents except sodium dantrolene (Norwich Eaton Pharmaceuticals, Norwich, NY) were from Sigma Chemical Co. (St. Louis, MO). Reduced $\mathrm{Ca}^{2+}$ medium consisted of $\mathrm{HL}-15$ in which $\mathrm{CaCl}_{2}$ was replaced with $\mathrm{MgCl}_{2}$; this medium likely contains at least $200 \mu \mathrm{M}$ $\mathrm{Ca}^{2+}$, originally present as contamination (Raaflaub, 1956). Zero $\mathrm{Ca}^{2+}$ medium consisted of HL-15 in which $\mathrm{CaCl}_{2}$ was replaced by $\mathrm{MgCl}_{2}$ with added $1 \mathrm{~mm}$ EGTA. Cells were incubated in a room air atmosphere at $22-25^{\circ} \mathrm{C}$. Medium changes were performed by carefully removing all but a thin film of medium and then gently adding fresh medium to the dishes. Growth cones were isolated from neurons with the aid of an electrolytically sharpened tungsten microknife; neurites were cut just proximal to the growth cone.

Assessments of neurite elongation rate and growth cone morphology. Cells and isolated neurites were examined and photographed on a Nikon Diaphot inverted microscope with phase-contrast optics. Two parameters of neurite outgrowth were assessed: neurite elongation and growth cone structures associated with motility (filopodia). Neurite elongation rates were quantified using tracings made from projected negatives of photographs taken sequentially. Linear-regression analysis of pre- and posttreatment elongation rates was used to obtain the post/pretreatment ratio, which is expressed as the percentage of the initial elongation rate. Growth cones were also examined on the projected photographic negatives; the number of filopodia/growth cone was used as index of motility since these structures are known to reflect the underlying activity of the growth cone cytoskeleton (Bray and Gilbert, 1981). Student's $t$ test was used for all statistical comparisons, and values are expressed as mean and SEM.

\section{Results}

Effects of elevations or reductions in $\mathrm{Ca}^{2+}$ influx on neurite elongation and growth cone morphology

Previous measurements of intracellular $\mathrm{Ca}^{2+}$ using fura-2 indicated that the intracellular $\mathrm{Ca}^{2+}$ level is maintained within a narrow range that is optimal for outgrowth; outgrowth was suppressed in neurons in which intracellular $\mathrm{Ca}^{2+}$ was increased by exposure to neurotransmitters or electrical activity, while neurons that stopped growing spontaneously had significantly lower levels of $\mathrm{Ca}^{2+}$ within their neurite tips (Cohan et al., 1986, 1987).

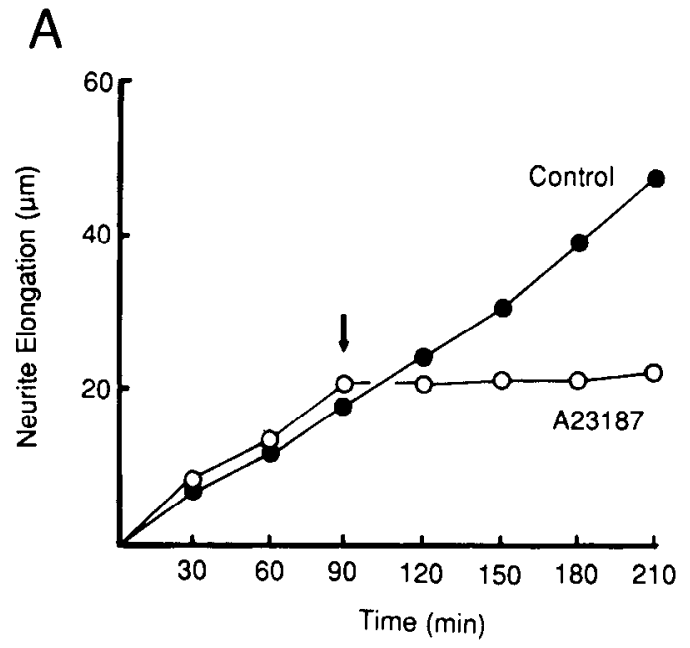

B

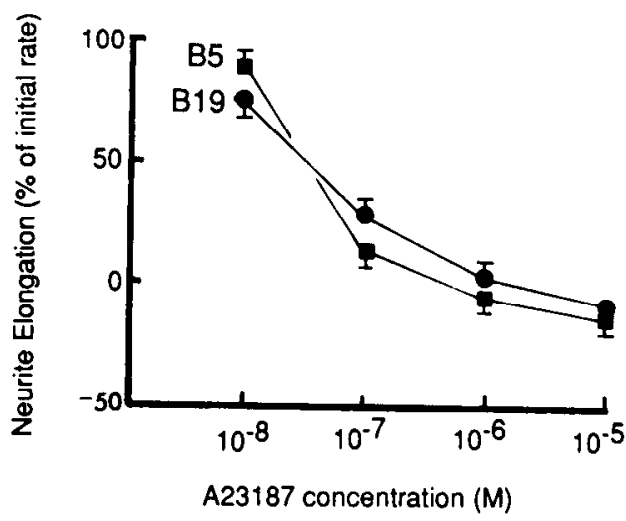

Figure 1. Time course and dose-dependence for inhibition of neurite elongation by A23187. $A$, Representative time course of A23187 suppression of neurite elongation. Neurons B5 and B19 were preincubated for $90 \mathrm{~min}$, followed by a $2 \mathrm{hr}$ incubation in the absence (Control) or presence of $10^{-7}$ M A23187 (arrow, addition of A23187). Examples shown are representative of all 33 neurites assessed. $B$, Following a 90 min preincubation to determine the initial ratc of ncurite elongation, neurons B19 and B5 were incubated $120 \mathrm{~min}$ in the presence of the indicated concentrations of ionophore A23187. Values are expressed as percentages of the initial elongation rate and represent the means and SEM of determinations on 11-22 neurites from 3-6 neurons. Values for A23187 at concentrations of $10^{-7} \mathrm{M}$ or greater were significantly different from control values $(p<0.01-0.0001)$, which averaged $101.0 \pm$ $4.6 \%$ of the initial rate.

In all cases in which neurite outgrowth was suppressed, the neurite tips were devoid of filopodia and lamellipodia, suggesting that growth cone movements and neurite elongation are associated processes. To causally relate the degree of $\mathrm{Ca}^{2+}$ influx from the cell exterior to changes in neurite elongation and growth cone movements, we assessed outgrowth after exposing neurons to manipulations known either to increase (A23187; Reed and Lardy, 1972) or reduce ( $\mathrm{Ca}^{2+}$ channel blockers; Weiss, 1974; Hagiwara and Byerly, 1981; reduced or zero $\mathrm{Ca}^{2+}$ medium) $\mathrm{Ca}^{2+}$ influx. Further, we titrated these treatments to determine whether the elongation and growth cone movement components of outgrowth had differential sensitivities to $\mathrm{Ca}^{2+}$. 

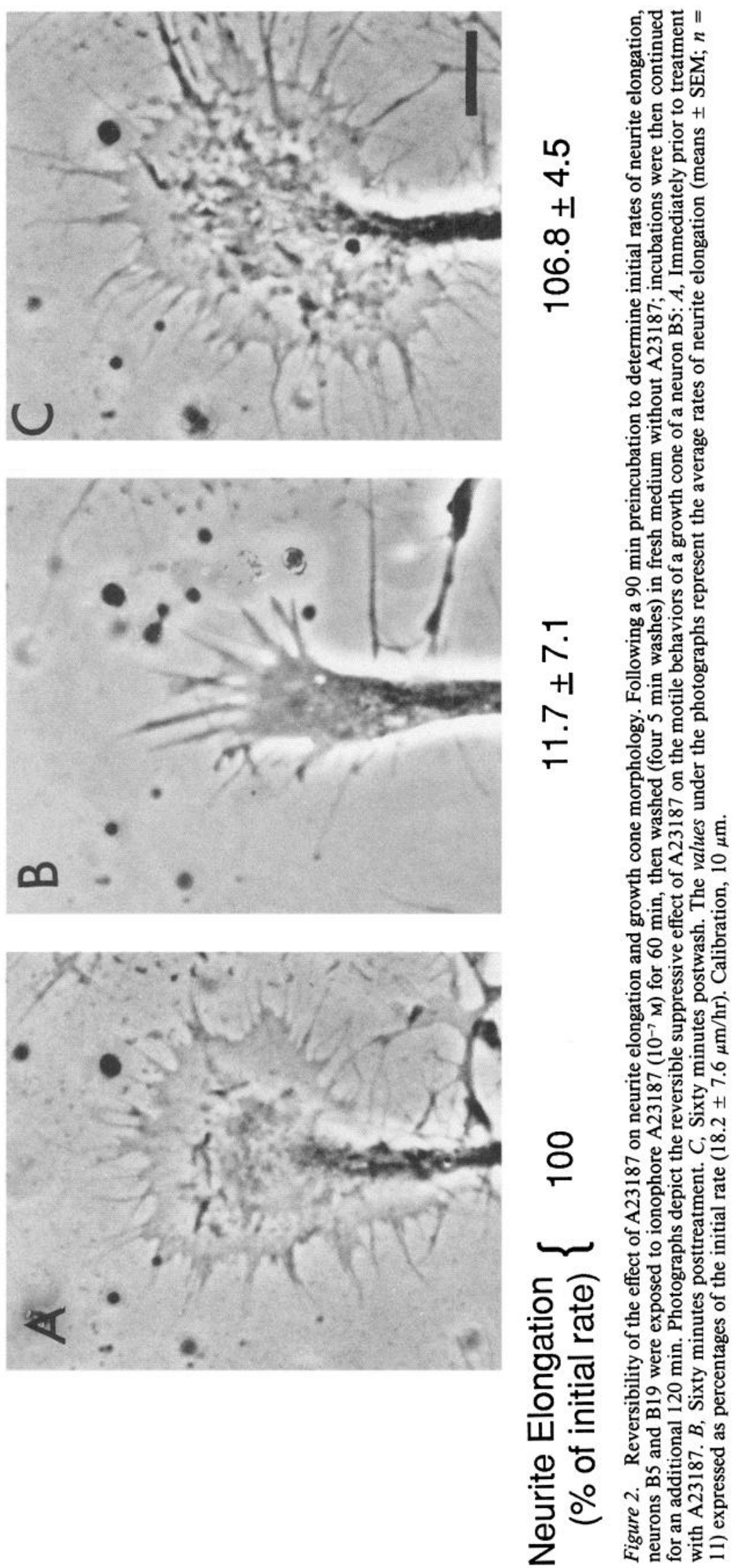

N

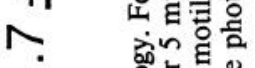

드 응호웜

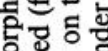

实市

उले

1

.

용

战它

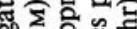

ㅇํㅇํㄹ

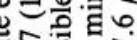

这

c

응

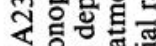

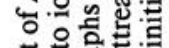

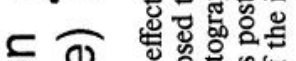

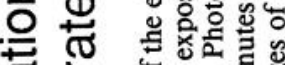

ชิ

으 을

W.

(1)

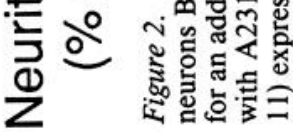



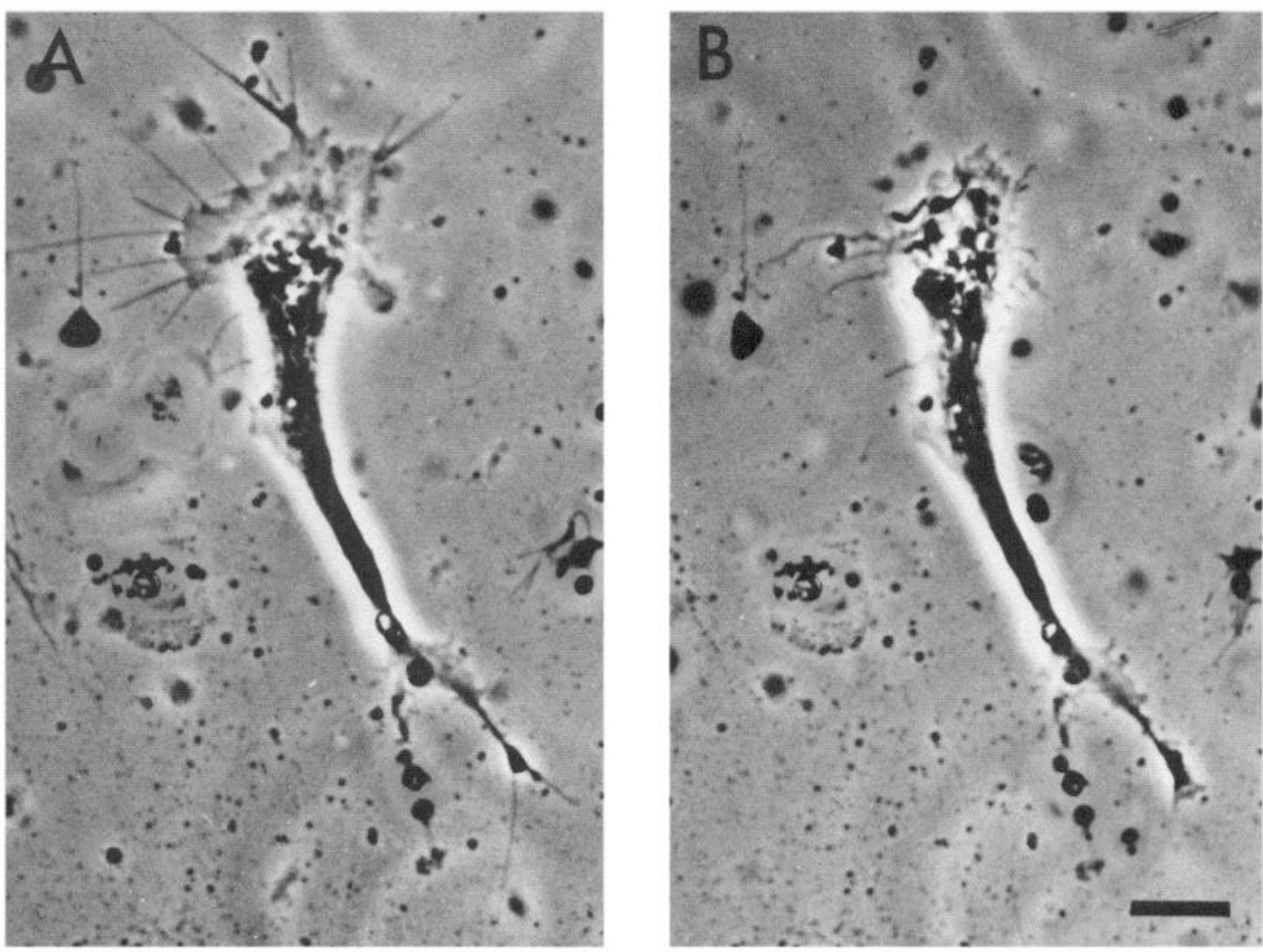

Figure 3. Effects of A23187 on the morphology of isolated growth cones. A, Photograph of an isolated growth cone from a neuron B5 taken 60 min after isolation and immediately prior to treatment with A23187; note the numerous filopodia and relatively broad lamellipodia. $B$, The same isolated growth cone as in $A, 15$ min following treatment with $10^{-7}$ M A23187; note the reduction in filopodial numbers and lamellipodial span. Calibration, $25 \mu \mathrm{m}$.

Effects of A23187 on neurite elongation and growth cone morphology. Neurite elongation was reduced to near zero within 30 min of treatment with A23187 at $10^{-7} \mathrm{M}$; the suppressive effect was dose-dependent for A23187 concentrations from $10^{-8}$ to $10^{-5} \mathrm{M}$ (Fig. 1). Neurite retraction occurred at the highest A23187 concentration of $10^{-5} \mathrm{M}$. The $\mathrm{ED}_{50}$ for suppression was approximately $6 \times 10^{-8} \mathrm{M}$. The suppressive effect of A23187 on neurite elongation was reversible; following suppression by $10^{-7} \mathrm{M}$ A23187, neurons were washed with fresh medium, and the neurite elongation rate returned within $60 \mathrm{~min}$ to the pretreatment level (Fig. 2). This reversibility was not simply the result of the medium change; when A23187 was included in the postwash medium, elongation remained suppressed (cf. Fig. 6).

The expression of growth cone motility can be described morphologically. Motile growth cones are flat and protrude numerous filopodia (Fig. 2A). In contrast, nonmotile growth cones generally assume a rounded or clubbed appearance, and have greatly reduced numbers of filopodia (Fig. 2B). The suppression of elongation caused by A23187 at all effective concentrations was accompanied by retraction of growth cone filopodia and lamellipodia, resulting in a clubbed neurite tip (Fig. $2 B$ ) similar to that accompanying spontaneous, or neurotransmitter or electrically induced, cessation of outgrowth (Haydon et al., 1984; Cohan and Kater, 1986). A23187 caused significant reductions in the numbers of filopodia on growth cones of both neurons B19 and B5 (Table 1). These morphological changes were completely reversed following washout of the ionophore as growth cone filopodia and lamellipodia reappeared and elongation resumed (Fig. 2). The temporal relationship of reductions in growth cone motility and neurite elongation was such that elongation ceased within $30 \mathrm{~min}$ of exposure to A23187 and before all filopodia had retracted (Fig. 2). In most cases ( 8 of 10 neurites examined) no filopodia were evident by 90 min posttreatment.

To determine whether the suppression of growth cone movements caused by A 23187 was exerted locally on the growth cones or required mediation by the soma, we tested the effects of A23187 on isolated growth cones. A23187 (10-7 M) stopped elongation of isolated growth cones (the rate was reduced to near zero in 11 of 11 isolated growth cones). Furthermore, A23187 caused the retraction of filopodia and lamellipodia in isolated growth cones (Fig. 3). Thus, increased $\mathrm{Ca}^{2+}$ influx can act locally to suppress both neurite elongation and growth cone movements.

Table 1. Effects of $\mathrm{A23187}$ and $\mathrm{La}^{3+}$ upon growth cone filopodia

\begin{tabular}{llcl} 
& & \multicolumn{2}{l}{ Filopodia/growth cone } \\
\cline { 3 - 4 } Treatment & Neuron & $\begin{array}{l}\text { Before } \\
\text { treatment }\end{array}$ & $\begin{array}{l}\text { After } \\
\text { treatment }\end{array}$ \\
\hline $\mathrm{A} 23187\left(10^{-7} \mathrm{M}\right)$ & B5 & $21.8 \pm 2.3^{a}$ & $3.7 \pm 0.9^{*}$ \\
& B19 & $8.8 \pm 0.6$ & $0.8 \pm 0.3^{*}$ \\
$\mathrm{La}^{3+}\left(10^{-5} \mathrm{M}\right)$ & B5 & $16.4 \pm 1.5$ & $1.2 \pm 0.4^{*}$ \\
& B19 & $5.7 \pm 0.6$ & $0.5 \pm 0.2^{*}$ \\
\hline
\end{tabular}

Values represent the means \pm SEM of determinations on 10 growth cones. Filopodial counts were made immediately prior to treatment and $60 \mathrm{~min}$ after treatment.

${ }^{a}$ Growth cones of neurons B5 possess significantly more filopodia than do B19 growth cones ( $p<0.05$; cf. Haydon et al., 1985).

${ }^{*} p<0.001$, compared to before-treatment values. 
Figure 4. Effects of $\mathrm{La}^{3+}$ on growth cone morphology. A, A growing neuron B19 with active growth cones $(a-c)$ immediately prior to exposure to $\mathrm{LaCl}_{3}$ (final concentration, $10^{-5} \mathrm{M}$ ). $B$, The same neuron as in $A 60 \mathrm{~min}$ after exposure to $\mathrm{La}^{3+}$; growth cone filopodia and lamellipodia were reduced, but neurite elongation continued. Arrows point to varicosities present where growth cones existed prior to exposure to $\mathrm{La}^{3+}$. Drawings represent enlarged tracings of the growth cones of the 3 neurites $(a-c)$. The observations depicted by these photographs are representative of the morphological effects seen with $\mathrm{La}^{3+}$ on 35 of 37 neurites from 11 neurons. Calibration, $100 \mu \mathrm{m}$.
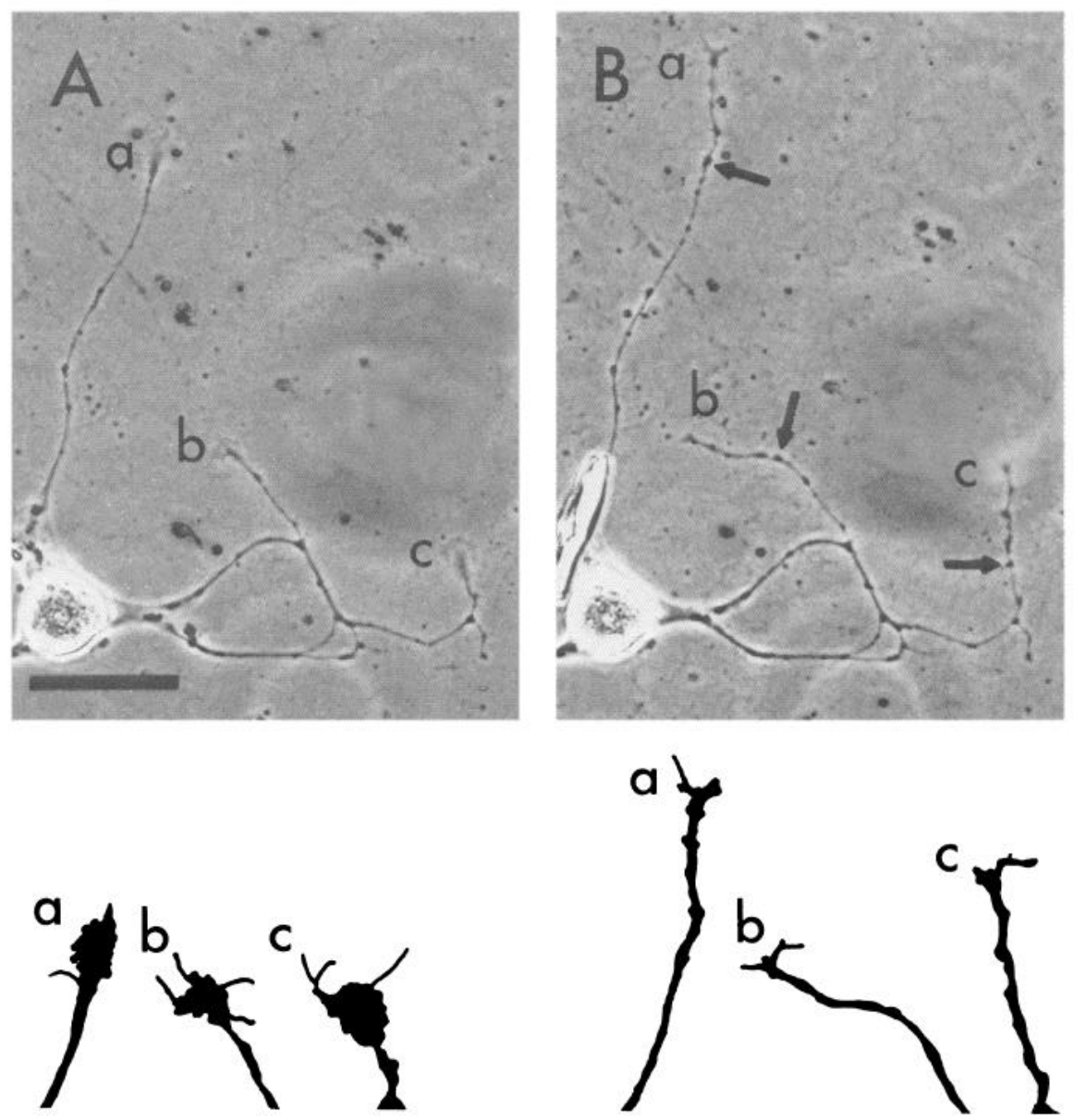

Effects of calcium channel blockers on neurite elongation and growth cone morphology. We exposed neurons to polyvalent cation $\mathrm{Ca}^{2+}$ channel blockers known to act on a broad spectrum of cell types $\left(\mathrm{La}^{3+}, \mathrm{Co}^{2+}, \mathrm{Cd}^{2+}\right.$; Weiss, 1974; Hagiwara and Byerly, 1981) and determined the dose-dependencies and specificities of their effects on neurite outgrowth. Consistent with the hypothesis that specific intracellular $\mathrm{Ca}^{2+}$ levels are optimal for normal outgrowth, we found that $\mathrm{La}^{3+}\left(10^{-4} \mathrm{M}\right), \mathrm{Co}^{2+}\left(10^{-2} \mathrm{M}\right)$, and $\mathrm{Cd}^{2+}\left(10^{-4} \mathrm{M}\right)$ all significantly suppressed neurite elongation (suppression to 51, 42, and $30 \%$ of control levels for $\mathrm{La}^{3+}, \mathrm{Co}^{2+}$, and $\mathrm{Cd}^{2+}$, respectively; $p<0.001-0.0001 ; n=8-20$ ). This suppression presumably resulted from a reduction in $\mathrm{Ca}^{2+}$ influx and possibly from a lowering of intracellular $\mathrm{Ca}^{2+}$ levels. The retraction of growth cone filopodia and lamellipodia was coincident with suppression of elongation. Organic blockers of $\mathrm{Ca}^{2+}$ influx from the cell exterior (nifedipine and verapamil; Hagiwara and Byerly, 1981) and a blocker of release from intracellular stores (dantrolene; Van Winkle, 1976) were without effect at concentrations expected from other systems to block calcium fluxes (12-19 neurites, assessed from 3-5 neurons/treatment). This set of results further supports the hypothesis that neurite outgrowth is promoted only when $\mathrm{Ca}^{2+}$ influx is at a specific level required for outgrowth. However, further experiments, described below, indicate that the elongation and growth cone movement components of outgrowth appear to have somewhat different $\mathrm{Ca}^{2+}$ sensitivities.

Previous studies using cytoskeleton-altering drugs showed that elongation can occur in neurites devoid of filopodial and la- mellipodial growth cone movements (Marsh and Letourneau, 1984; Letourneau, 1985). We observed a similar phenomenon when neurons were treated with lower concentrations of $\mathrm{Ca}^{2+}$ channel blockers than were used above $\left(\mathrm{La}^{3+}, 10^{-5} \mathrm{M} ; \mathrm{Co}^{2+}, 10^{-3}\right.$ M; $\mathrm{Cd}^{2+}, 5 \times 10^{-5} \mathrm{M}$; Fig. 4). At these concentrations growth cone filopodia were significantly reduced (Table 1), but neurite elongation continued and was, in fact, accelerated (elongation rate was increased to 153,182 , and $123 \%$ of controls for $\mathrm{La}^{3+}$, $\mathrm{Co}^{2+}$, and $\mathrm{Cd}^{2+}$, respectively; $\left.p<0.05-0.001 ; n=9-24\right)$. These low concentrations of $\mathrm{Ca}^{2+}$ blockers had similar effects on the elongation rates and morphology of isolated growth cones (not shown). Branching was not observed in neurites elongating in response to $\mathrm{La}^{3+}$ ( 35 neurites monitored for $4 \mathrm{hr}$ following exposure to $\mathrm{La}^{3+}$ ), while, under the same experimental protocol, nearly $50 \%$ ( 21 of 55 ) control neurites branched. Thus, given the appropriate status of cellular $\mathrm{Ca}^{2+}$, neurites could elongate without filopodial growth cone movements, a condition that would greatly alter final neuronal architecture.

\section{$\mathrm{La}^{3+}$ blocks serotonin suppression of neurite elongation}

From 2 independent studies we knew that serotonin increases intracellular $\mathrm{Ca}^{2+}$, on the one hand (Cohan et al., 1986, 1987), and suppresses neurite outgrowth on the other hand (Haydon et al., 1984). To determine whether serotonin inhibition is mediated by $\mathrm{Ca}^{2+}$ influx, we tested the effects of low levels of the $\mathrm{Ca}^{2+}$ channel blocker $\mathrm{La}^{3+}$ on neurite outgrowth from neurons B19 before and after exposure to serotonin (Fig. 5). Prior application of $\mathrm{La}^{3+}\left(10^{-5} \mathrm{M}\right)$ prevented the normal suppressive 
response of the neurotransmitter and, as reported above, significantly accelerated neurite outgrowth to $150-180 \%$ of the base rate $(p<0.05-0.01)$. Of major interest in the regulation of neurite outgrowth is the degree to which neurons that have stopped growing can resume neurite outgrowth. Neurites inhibited by serotonin could be induced to grow again by exposure to $\mathrm{La}^{3+}$. In each of the 10 cases examined, neurites whose elongation and growth cone motility were completely suppressed by scrotonin recovered and rencwed clongation, though this was not true of growth cone motility following exposure to $\mathrm{La}^{3+}$ (Fig. 5). The morphology of these disinhibited neurites was indistinguishable from that seen for neurons treated with $\mathrm{La}^{3+}$ alone (compare Figs. 4 and 5); filopodial and lamellipodial structures were not observed in growth cones of $\mathrm{La}^{3+}$-treated neurons, and the neurite tips extended as narrow (neurite-width) processes (Fig. 5).

\section{Effects of reduced and zero extracellular calcium on neurite outgrowth: Interactions with serotonin, A23187, and $\mathrm{La}^{3+}$}

As a further test of the basic hypothesis of this study, we exposed neurons to media with either reduced $\left(\mathrm{MgCl}_{2}\right.$ substituted for $\left.\mathrm{CaCl}_{2}\right)$ or zero $\left(\mathrm{MgCl}_{2}\right.$ substituted for $\mathrm{CaCl}_{2}$ plus EGTA) $\mathrm{Ca}^{2+}$ and determined neurite elongation rates in the absence (controls) or presence of serotonin, A23187, or low levels of $\mathrm{La}^{3+}$. Before performing these experiments, it was necessary to determine several baseline data. Previous studies indicated that medium changes can accelerate neurite outgrowth (D. P. McCobb, personal communication); we therefore used as controls neurons that were washed in fresh HL-15 of normal $\mathrm{Ca}^{2+}$ composition. As expected, washing significantly increased the rate of neurite elongation to what we designate as the "control" level, which was $140 \pm 11 \%$ of the base rate $(p<0.005$; Fig. 6). The medium change had no obvious effects on growth cone lamellipodial or filopodial movements. Incubation in reduced $\mathrm{Ca}^{2+}$ medium caused a reduction in growth cone filopodia and lamellipodia (not shown) and increased the rate of neurite elongation to $140 \%$ of controls ( $p<0.05$; Fig. 6 ). Using this same paradigm, it was found that in normal $\mathrm{Ca}^{2+}$ medium, $\mathrm{La}^{3+}$ significantly stimulated elongation to $160 \%$ of controls, while A23187 and serotonin significantly suppressed elongation rates to 28 and $35 \%$ of controls, respectively (Fig. 6). With these data it was possible to directly test the extracellular $\mathrm{Ca}^{2+}$ requirement for the suppressive actions of serotonin and A23187. The key experiments were to pair treatments of serotonin or A23187 with the reduced extracellular calcium environment. In the normal $\mathrm{Ca}^{2+}$ environment, serotonin decreased the rate of neurite elongation to $28 \%$ of controls, while this same treatment in the reduced extracellular calcium environment had a significantly lesser suppressive effect (suppression to $70 \%$ of controls; Fig. 6). These results indicate that the rise in intracellular calcium recorded by fura- 2 measurements (Cohan et al., 1986, 1987) was indeed derived from calcium influxes from the extracellular space, and show that the influx is necessary for inhibition of neurite outgrowth. Indeed, a repeat of this experiment with A23187, a facilitator of $\mathrm{Ca}^{2+}$ influx, gave precisely the same results: The usual inhibitory effect of the ionophore (suppression of elongation to $35 \%$ of control levels) was not seen in the reduced extracellular calcium environment (Fig. 6). Thus, agents that suppress neurite outgrowth require extracellular $\mathrm{Ca}^{2+}$, presumably for influx.

The results to this point showed that expected increases (using A23187 treatment) or large decreases (using high concentrations of channel blockers) in cellular $\mathrm{Ca}^{2+}$ are suppressive to both neurite elongation and growth cone motility. Since decreasing extracellular $\mathrm{Ca}^{2+}$ or moderate blockage of $\mathrm{Ca}^{2+}$ influx with low concentrations of channel blockers can increase the rate of neurite elongation, it was of interest to test the effects on neurite elongation of these 2 treatments combined. Together, these treatments resulted in a significant reduction in the rate of elongation, compared to the rates for neurons exposed to either trcatment alone (Fig. 6). These results are in line with the concentration-dependent effects of $\mathrm{Ca}^{2+}$ channel blockers on neurite elongation rates. Thus, this dual treatment likely reduced $\mathrm{Ca}^{2+}$ influx below the critical level that allows for maximal rates of neurite elongation. Furthermore, the latter result suggests that a basal level of $\mathrm{Ca}^{2+}$ "leak" into the cell is required for neurite outgrowth. In support of this interpretation are the results of a final experiment in which cultured neurons were exposed to a true zero $\mathrm{Ca}^{2+}$ medium (containing $1 \mathrm{~mm}$ EGTA). In this environment the neurite elongation rate was reduced to $32 \pm$ $18.4 \%$ of the initial rate $(n=7)$.

\section{Discussion}

The present study has provided support for the hypothesis that changes in $\mathrm{Ca}^{2+}$ influx from the cell exterior can regulate 2 basic components of outgrowth, neurite elongation and growth cone movements. Furthermore, it appears that these 2 components have somewhat different $\mathrm{Ca}^{2+}$ requirements. It is important to emphasize that this hypothesis is based on evidence from 2 different approaches. Previous work strongly correlated $\mathrm{Ca}^{2+}$ levels within the growth cone with the growth status of the neuron (Cohan et al., 1986, 1987; Connor, 1986). The present study, on the other hand, examined the effects on neurite elongation and growth conc bchavior of a battery of agents known to change transmembrane $\mathrm{Ca}^{2+}$ movements. Both lines of evidence produced results entirely consistent with the above hypothesis.

We interpret the results of this study as indicating that cellular $\mathrm{Ca}^{2+}$ movements can be regulated over a range physiologically relevant to neurite outgrowth. During the usual outgrowth seen in cell culture, the intracellular $\mathrm{Ca}^{21}$ level is likely above that optimal for neurite elongation, but is at an optimum for growth cone filopodial and lamellipodial movements. Therefore, low concentrations of $\mathrm{Ca}^{2+}$ channel blockers that suppress growth cones but promote elongation probably decrease a constant "leak" $\mathrm{Ca}^{2+}$ current into the cell. In support of such a basal rate of $\mathrm{Ca}^{2+}$ influx are the data of Freeman et al. (1985), who used a vibrating probe to measure steady inward currents, thought to be $\mathrm{Ca}^{2+}$, at the growth cones of cultured fish retinal ganglion cells. Thus, by regulating the degree of $\mathrm{Ca}^{2+}$ influx, the outgrowth status of the neuron can be precisely tuned.

Several sets of data now suggest that a critical concentration of intracellular $\mathrm{Ca}^{2+}$ is necessary for normal outgrowth of neurites: (1) Increases in growth cone $\mathrm{Ca}^{2+}$ induced by serotonin or electrical activity (Cohan et al., 1986, 1987) or by A23187 (Figs. 1-3) caused a reversible inhibition of both neurite elongation and growth cone filopodial movements. (2) High concentrations of $\mathrm{Ca}^{2+}$ channel blockers also suppressed outgrowth. (3) Lower concentrations of $\mathrm{Ca}^{2+}$ channel blockers (and by inference a critical $\mathrm{Ca}^{2+}$ influx) simultaneously reduced growth cone movements and enhanced neurite elongation. (4) Combined exposure to treatments that independently increase outgrowth rates (i.e., reduced extracellular $\mathrm{Ca}^{2+}$ and $\mathrm{La}^{3+}$ ) did not enhance elongation rates (Fig. 6), indicating that a specific level of $\mathrm{Ca}^{2+}$ influx is 


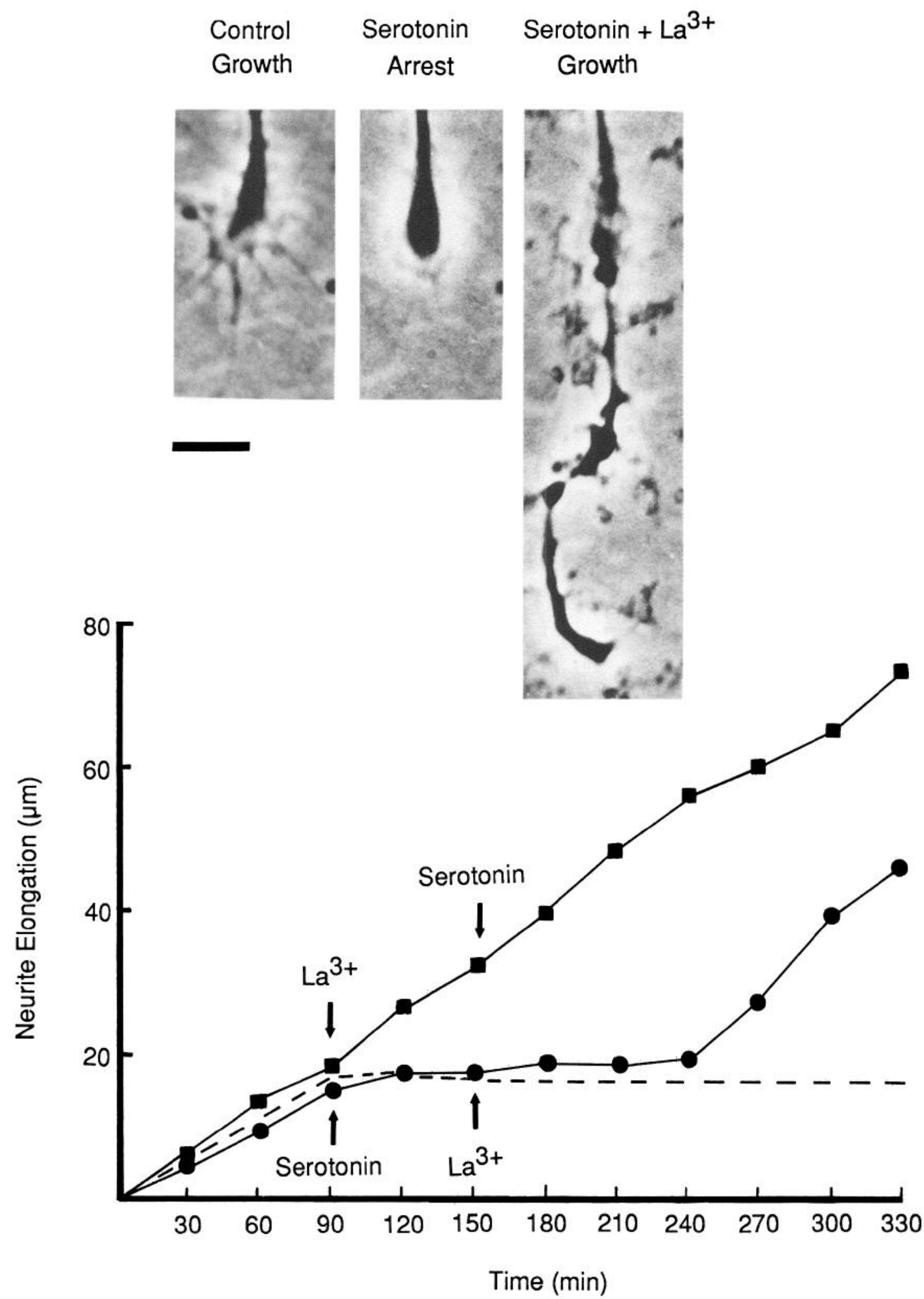

Figure 5. Effects of staggered $\mathrm{La}^{3+}$ and serotonin treatments on neurite elongation and growth cone morphology of neurons B19. Preincubation of $90 \mathrm{~min}$ was followed by addition of either $\mathrm{La}^{3+}$ or serotonin $\left(10^{-5}\right.$ and $10^{-4} \mathrm{M}$, respectively), as indicated by the arrows. The dashed line represents the average time course of elongation of neurites $(n=7)$ from a neuron B19 exposed to serotonin at the 90 min time point; note that suppression continues throughout the time course of the experiment. For combined treatments (solid lines), the points are from individual neurites and are representative of $5\left(\mathrm{La}^{3+}\right.$ treatment followed by serotonin) or 10 (serotonin treatment followed by $\left.\mathrm{La}^{3+}\right)$ cases. Statistical comparisons (values are expressed as percentages of the initial elongation rate, which averaged $11.8 \pm 3.4 \mu \mathrm{m} / \mathrm{hr}$ ) are as follows: For consecutive treatments with $\mathrm{La}^{3+}$ and serotonin, the rates were $155.2 \pm 10.3 \%$ during exposure to $\mathrm{La}^{3+}$ prior to serotonin $(p<0.005$ as compared to the initial rate) and $179.4 \pm 15.9 \%$ following serotonin $\left(p<0.005\right.$ as compared to the initial rate). For consecutive treatments with serotonin and $\mathrm{La}^{3+}$, the rates were $31.3 \pm 5.9 \%$ 


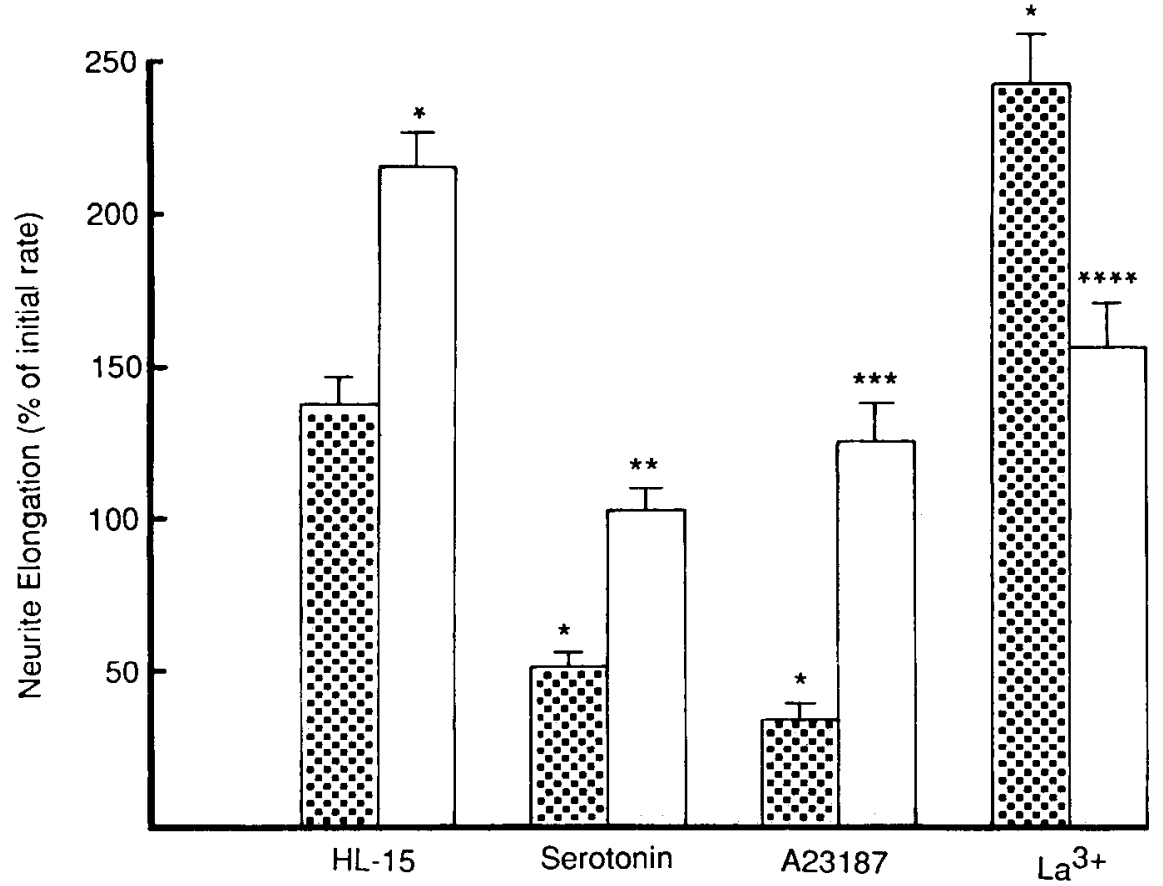

Figure 6. Effects on neurite elongation of serotonin, $\mathrm{La}^{3+}$, and $\mathrm{A} 23187$ in relation to extracellular $\mathrm{Ca}^{2+}$. Elongation rates of neurites from neurons B19 and B5 (serotonin experiments were performed on neurons B19) were determined before and after replaccment of medium with fresh HL-15 containing either physiological levels of $\mathrm{Ca}^{2+}$ (4.1 $\mathrm{mM} \mathrm{CaCl}_{2}$, stippled bars) or reduced $\mathrm{CaCl}_{2} \mathrm{HL}-15$ (open bars), with or without the following agents: serotonin $\left(10^{-4} \mathrm{M}\right), \mathrm{A} 23187\left(10^{-7} \mathrm{M}\right)$, or $\mathrm{La}^{3+}$ $\left(10^{-5} \mathrm{M}\right)$. Values represent the means and SEM of measurements on 11-18 neurites from 3-4 neurons/treatment group. ${ }^{*}, p<0.01-0.0001$, as compared to $\mathrm{HL}-15$, plus $\mathrm{Ca}^{2+}$. ${ }^{* *}, p<0.01$, as compared to $\mathrm{HL}-15$, minus $\mathrm{Ca}^{2+} ; p<$ 0.001 as compared to serotonin, plus $\mathrm{Ca}^{2+}$. ${ }^{* * *}, p<0.01$, comparcd to $\mathrm{HL}$ 15 , minus $\mathrm{Ca}^{2+} ; p<0.001$, as compared to $\mathrm{A} 23187$, plus $\mathrm{Ca}^{2+} .{ }^{* * * *}, p<0.05$, compared to $\mathrm{HL}-15$, minus $\mathrm{Ca}^{2+} ; p<$ 0.05 , compared to $\mathrm{La}^{3+}$, plus $\mathrm{Ca}^{2+}$. required for a maximum rate of outgrowth. Furthermore, the finding that removal of all extracellular $\mathrm{Ca}^{2+}$ with EGTA suppressed outgrowth indicates that some basal rate of $\mathrm{Ca}^{2+}$ movement into the neuron is required to maintain outgrowth (i.e., an equilibrium with cellular $\mathrm{Ca}^{2+}$ reduction mechanisms).

Although $\mathrm{Ca}^{2+}$ appears to be a general regulator of neurite outgrowth, it is also clear that responses depend on both the cell type studied and the experimental conditions employed. While previous investigators have used some of the agents we employed in the present study to test $\mathrm{Ca}^{2+}$ involvement in outgrowth regulation (Schubert et al., 1978; Gundersen and Barrett, 1980; Anglister et al., 1982; Kostenko et al., 1983; Bixby and Spitzer, 1984; Reboulleau, 1986), their studies did not use the full spectrum or the titrations of agents used here, and produced rather confounding and apparently contradictory results. For example, Reboulleau (1986) found that ionophore A23187 promoted neurite outgrowth in a rat CNS neuroblastoma cell line. While this result suggests that increases in $\mathrm{Ca}^{2+}$ influx promote outgrowth, our interpretation, based on the data of the present study, is that rates of $\mathrm{Ca}^{2+}$ influx prior to treatment in that particular system were below levels optimum for neurite outgrowth. Similarly, Gundersen and Barrett (1980) found that cultured chick dorsal root ganglion neurites turned towards a $\mathrm{Ca}^{2+}$ source when ionophore A23187 was included in the incubation medium. Quite different results were obtained in normal vertebrate (Letourneau and Wessells, 1974; Bixby and Spitzer, 1984) and molluscan (Kostenko et al., 1983; Cohan et al., 1987; and the present study) CNS neurons, where agents expected to increase $\mathrm{Ca}^{2+}$ influx inhibited neurite outgrowth. In contrast, in several neuronal tumor cell lines (Schubert et al., 1978; Anglister et al., 1982; Reboulleau, 1986) and in cultured chick retinal neurons (Suarez-Isla et al., 1984) $\mathrm{Ca}^{2+}$ influx promoted outgrowth. Of particular interest in relation to our data is the work by Bixby and Spitzer (1984), who reported a stimulation of neurite elongation rate in embryonic frog neurons by medium lacking $\mathrm{Ca}^{2+}$ and containing EGTA (growth cones were not examined). The latter result can be explained if one considers that $\mathrm{Ca}^{2+}$ influx rates in the cases of both frog embryonic and Helisoma neurons are above those optimal for neurite elongation, with the levels in the frog neurons likely being considerably above optimum than those in Helisoma.

The variety of results described above indicates that different neuronal types, examined under different environmental conditions, may have basal levels of intracellular $\mathrm{Ca}^{2+}$ either below, above, or within the range optimal for neurite elongation and/ or growth cone movements. By considering the previous work in this context, it is possible to reconcile most of the observations made on $\mathrm{Ca}^{2+}$ and neurite outgrowth. This can be done by defining the basal level of intracellular $\mathrm{Ca}^{2+}$ in the neuron under study as the "Ca ${ }^{2+}$ set-point." For example, the $\mathrm{Ca}^{2+}$ set-point of Helisoma neurons in the present study was above the optimum level for neurite elongation but within the optimum range for growth cone movements. Thus, manipulations expected to increase $\mathrm{Ca}^{2+}$ influx (i.e., added A23187) suppressed both elongation and growth cone motility, while exposure to conditions expected to cause moderate reductions in calcium influx (low levels of $\mathrm{Ca}^{2+}$ channel blockers, reduced $\mathrm{Ca}^{2+}$ medium) suppressed growth cone motility and promoted neurite elongation.

for the $2.5 \mathrm{hr}$ interval following exposure to serotonin $(p<0.001$, compared to the initial rate) and $155.6 \pm 22.0 \%$ for the time points after 240 $\min (p<0.001$, compared to the rate during serotonin suppression). Micrographs are of a growth cone immediately prior to serotonin treatment (control growth), $60 \mathrm{~min}$ after exposure to $10^{-4} \mathrm{M}$ serotonin and immediately prior to treatment with $\mathrm{La}^{3+}$ (serotonin arrest), and $3 \mathrm{hr}$ after exposure to $10^{-5} \mathrm{M} \mathrm{LaCl}_{3}$ (in the continued presence of serotonin). These effects were seen in all of 10 neurites from 6 neurons examined. Calibration, $25 \mu \mathrm{m}$. 


\section{Calcium channels and neurite outgrowth}

A series of findings suggests that the effects on neurite outgrowth of multivalent cation blockers seen in Helisoma neurons are due to specific effects on $\mathrm{Ca}^{2+}$ channels: (1) We observed the following order of efficacy of $\mathrm{Ca}^{2+}$ blockers on neurite outgrowth: $\mathrm{La}^{3+}>\mathrm{Cd}^{2+}>\mathrm{Co}^{2+}$, which was consistent with their order of potency in blocking $\mathrm{Ca}^{2+}$ currents and with their binding affinity in several invertebrate muscle and nerve preparations (Klee et al., 1973; Kostyuk et al., 1977). The lack of effect of organic blockers on neurite outgrowth is consistent with their relative lack of effect on calcium currents in other molluscan cells (Klee et al., 1973; Kostyuk et al., 1977); likewise the lack of effect of dantrolene, a blocker of calcium release from intracellular stores, on basal or serotonin-inhibited outgrowth is consistent with the plasma membrane $\mathrm{Ca}^{2+}$ channels being primary sites where serotonin acts to induce $\mathrm{Ca}^{2+}$ influx. (2) $\mathrm{Co}^{2+}$ blocked action potential-induced influx of $\mathrm{Ca}^{2+}$ into neurons B19 and B5 (Cohan et al., 1987). (3) Only a narrow concentration range of inorganic blockers induced neurite elongation. (4) Neurons incubated in reduced $\mathrm{Ca}^{2+}$ medium showed outgrowth behaviors (accelerated elongation and reduced growth cone movements) similar to those seen in neurons exposed to low levels of $\mathrm{Ca}^{2+}$ channel blockers. Furthermore, our findings indicate that a major locus of action of scrotonin in increasing growth cone $\mathrm{Ca}^{2+}$ levels is at the entry of extracellular $\mathrm{Ca}^{2+}$, rather than on release from intracellular stores. We found that under conditions of low levels of $\mathrm{Ca}^{2+}$ channel blockers or reduced extracellular $\mathrm{Ca}^{2+}$, serotonin was less effective in inhibiting neurite outgrowth from responsive Helisoma neurons. It thus appears that serotonin acts to suppress outgrowth by activating intracellular events that ultimately open plasma membrane $\mathrm{Ca}^{2+}$ channels.

To fully understand how changes in influx result in changes in intracellular $\mathrm{Ca}^{2+}$, we must also identify those mechanisms responsible for sequestering or buffering $\mathrm{Ca}^{2+}$, as well as those for gating $\mathrm{Ca}^{2+}$ currents. In any case, it is clear from our data and from previous fura-2 studies (Cohan et al., 1986, 1987) that agents like serotonin induce increases in intracellular $\mathrm{Ca}^{2+}$ by increasing influx through the plasma membrane.

\section{Calcium as a regulator of neuronal architecture}

When and where a neurite branches during its development ultimately determincs major aspects of neuronal form. In cell culture, where the process of branching is most readily studied, a branch point is seen as a region of splitting for an advancing growth cone. Thus, in the present study, under conditions expected to reduce $\mathrm{Ca}^{2+}$ influx, which allowed neurite elongation in the absence of growth cone filopodia and lamellipodia, it followed that branching failed to occur. These results are of obvious importance to the iteration of neuronal form and appear not to be an artifact of nonspecific effects of $\mathrm{Ca}^{2+}$ channel blockers or reduced $\mathrm{Ca}^{2+}$ medium. Similar effects have been observed in 2 other noteworthy cases. Vertebrate sympathetic neurons in culture were induced to grow in the absence of growth cone filopodia and lamellipodia; this was achieved by combined exposure to a microfilament-disrupting drug and an agent that promotes microtubule polymerization (Letourneau, 1985). Thus, elongation in the absence of growth cones is not a unique response of Helisoma neurons to low levels of $\mathrm{Ca}^{2+}$ channel blockers or reduced $\mathrm{Ca}^{2+}$ medium. In a study more directly related to normal neuronal development, LoPresti et al. (1973) reported that during the development of Daphnia in situ, only a few neuronal axons possessed growth cones, while others elongated without apparent growth cone structures. Taken together with these reports, our results lead to the conclusion that the cellular machineries underlying growth cone movements and neurite elongation have quite different roles in generating neuronal architecture. The filopodia and lamellipodia of growth cones are active in pathfinding and neurite branching, while other, relatively independent, cytoskeletal events associated with the neuritic shaft act to bring about elongation. Given this partitioning of roles and the differentiation of these 2 components of neurite outgrowth by treatments expected to influence influx of $\mathrm{Ca}^{2+}$, it seems clear that neurons may regulate their form by carefully controlling cellular $\mathrm{Ca}^{2+}$ movements. A neuron may respond to environmental cues by growth, growth and branching, turning, or stopping by regulating $\mathrm{Ca}^{2+}$ movements within different regions of the neurite.

\section{References}

Adelstein, R. S., and E. Eisenberg (1980) Regulation and kinetics of the actin-myosin-ATP interaction. Annu. Rev. Biochem. 49: 921956.

Anglister, L., I. C. Farber, A. Shahar, and A. Grinvald (1982) Localization of calcium sensitive calcium channels along developing neurites: Their possible role in regulating neurite elongation. Dev. Biol. 94: $351-365$.

Bixby, J. L., and N. C. Spitzer (1984) Early differentiation of vertebrate spinal neurons in the absence of voltage-dependent $\mathrm{Ca}^{2+}$ and $\mathrm{Na}^{+}$ influx. Dev. Biol. 106: 89-96.

Bray, D., and D. Gilbert (1981) Cytoskeletal elements in neurons. Annu. Rev. Neurosci. 4: 505-523.

Cohan, C. S., and S. B. Kater (1986) Suppression of neurite elongation and growth cone dynamics by electrical activity. Science $232: 1638-$ 1640 .

Cohan, C. S., J. A. Connor, and S. B. Kater (1986) Signals which inhibit growth cone motility also increase intracellular calcium levels within the growth cone. Soc. Neurosci. Abstr. 12: 370 .

Cohan, C. S., J. A. Connor, and S. B. Kater (1987) Electrically and chemically mediated increases in intracellular calcium in neuronal growth cones. J. Neurosci. 7: 3588-3599.

Connor, J. A. (1986) Digital imaging of free calcium changes and of spatial gradients in growing processes in single, mammalian central nervous system cells. Proc. Natl. Acad. Sci. USA 83: 6179-6183.

Freeman, J. A., P. B. Manis, G. J. Snipes, B. N. Mayes, P. C. Samson, J. P. Wikswo, and D. B. Freeman (1985) Steady growth cone currents revealed by a novel circularly vibrating probe: A possible mechanism underlying neurite growth. J. Neurosci. Res. 13: 257-283.

Greene, L. A., and E. M. Shooter (1980) The nerve growth factor. Annu. Rev. Neurosci. 4: 353-402.

Gundersen, R. W., and J. N. Barrett (1980) Characterization of the turning response of dorsal root neurites toward nerve growth factor. J. Cell Biol. 87: 546-554.

Hagiwara, S., and L. Byerly (1981) Calcium channel. Annu. Rev. Neurosci. 4: 69-125.

Hama, T., K. P. Huang, and G. Guroff (1986) Protein kinase C as a component of a nerve growth factor-sensitive phosphorylation system in PC12 cells. Proc. Natl. Acad. Sci. USA 83: 2353-2357.

Haydon, P.G., D. P. McCobb, and S. B. Kater (1984) Serotonin selectively inhibits growth cone dynamics and synaptogenesis of specific identified neurons. Science 226: 561-564.

Haydon, P. G., C. S. Cohan, D. P. McCobb, H. R. Miller, and S. B. Kater (1985) Neuron-specific growth cone properties as seen in identified neurons of Helisoma. J. Neurosci. Res. 13: 135-147.

Ishii, D. N. (1978) Effect of tumor promoters on the response of cultured embryonic chick ganglia to nerve growth factor. Cancer Res. 38: 3886-3893.

Kater, S. B. (1985) Dynamic regulators of neuronal form and connectivity in the adult snail Helisoma. In Model Neural Networks and Behavior, A. I. Selverston, ed., Plenum, New York.

Klee, M. R., K. C. Lee, and Y. Matsuda (1973) Interaction of D600 
and cobalt with the inward and outward current systems in Aplysia neurons. Pfluegers Arch. 343: R60.

Kostenko, M. A., V. S. Musienko, and T. I. Smolikhina (1983) $\mathrm{Ca}^{2+}$ and $\mathrm{pH}$ affect the neurite formation in cultured mollusc isolated neurons. Brain Res. 276: 43-50.

Kostyuk, P. G., O. A. Krishtal, and Y. A. Shakhovalov (1977) Separation of the sodium and calcium currents in the somatic membrane of mollusc neurons. J. Physiol. (Lond.) 270: 545-568.

Letourneau, P. C. (1985) Axonal growth and guidance. In Molecular Bases of Neural Development, G. M. Edelman, W. E. Gall, and W. M. Cowan, eds., pp. 269-294, Wiley, New York

Letourneau, P. C., and N. K. Wessells (1974) Migratory cell locomotion versus nerve axon elongation. Differences based on the effects of lanthanum ion. J. Cell Biol. 61: 56-69.

Levi-Montalcini, R., and P. U. Angeletti (1968) Nerve growth factor. Physiol. Rev. 48: 534-569.

Llinas, R. R. (1979) The Neurosciences: Fourth Study Program, F. O. Schmitt and F. G. Worden, eds., pp. 555-571, MIT Press, Cambridge, MA.

LoPresti, V., E. R. Macagno, and C. Levinthal (1973) Structure and development of neuronal connections in isogenic organisms: Cellular interactions in the development of the optic lamina of Daphnia. Proc. Natl. Acad. Sci. USA 70: 433-437.

Marsh, L., and P. C. Letourneau (1984) Growth of neurites without filopodial or lamellipodial activity in the presence of cytochalasin B. J. Cell Biol. 99: 2041-2047.

Nirenberg, M., S. Wilson, H. Higashida, A. Rotter, K. Krueger, N. Busis, R. Ray, J. G. Kenimer, and M. Adler (1984) Modulation of synapse formation by cyclic adenosine monophosphate. Science 222: 794799.

Raaflaub, J. (1956) Methods of Biochemical Analysis, Interscience, New York, pp. 301-325.
Reboulleau, C. P. (1986) Extracellular calcium-induced neuroblastoma differentiation: Involvement of phosphatidylinositol turnover. J. Neurochem. 46: 920-930.

Reed, P. W., and H. A. Lardy (1972) A23187: A divalent cation ionophore. J. Biol. Chem. 247: 6970-6977.

Schliwa, M., U. Euteneuer, J. C. Bulinski, and J. C. Izant (1981) Calcium lability of cytoplasmic microtubules and its modulation by microtubule-associated proteins. Proc. Natl. Acad. Sci. USA 78: $1037-$ 1041 .

Schubert, D., M. LaCorbiere, C. Whitlock, and W. Stallcup (1978) Alterations in the surface properties of cells responsive to nerve growth factor. Nature 273: 718-723.

Spinelli, W., and D. N. Ishii (1983) Tumor promoter receptor regulation of neurite formation in cultured human neuroblastoma cells. Cancer Res. 43: 4119-4125.

Suarez-Isla, B. A., D. J. Pelto, J. M. Thompson, and S. I. Rapoport (1984) Blockers of calcium permeability inhibit neurite extension and formation of neuromuscular synapses in cell culture. Dev. Brain Res. 14: 263-270.

Van Winkle, W. B. (1976) Calcium release from skeletal muscle sarcoplasmic reticulum: Site of action of dantrolene sodium? Science 193: 1130-1131.

Weiss, G. B. (1974) Cellular pharmacology of lanthanum. Annu. Rev. Pharmacol. 14: 343-354.

Wong, R. G., R. D. Hadley, S. B. Kater, and G. C. Hauser (1981) Neurite outgrowth in molluscan organ and cell cultures: The role of conditioning factor(s). J. Neurosci. 1: 1008-1021.

Wong, R. G., D. L. Barker, S. B. Kater, and D. A. Bodnar (1984) Nerve growth-promoting factor produced in culture media conditioned by specific CNS tissues of the snail Helisoma. Brain Res. 292: 81-91. 